\title{
A historical overview of traditional medicine practices and policy in Ethiopia
}

\author{
${ }^{1}$ Kebede Deribe Kassaye, ${ }^{1}$ Alemayehu Amberbir, ${ }^{1}$ Binyam Getachew, ${ }^{1}$ Yunis Mussema
}

\begin{abstract}
Background: Although traditional medicine plays an important role in Ethiopian society, knowledge about the extent and characteristics of traditional healing practices and practitioners is limited and has frequently been ignored in the national health system.

Objective: To review history of practices and policies on traditional medicine in Ethiopia.

Methods: A systematic review of available literature on Ethiopian traditional medicine and policy documents was carried out. Google, Pub Med and Medline online internet searches were done to access relevant material. In addition, materials from Jimma University Library and the Ethiopian Federal Ministry of Health Library were used. Several authorities were also contacted to supplement the literature.

Result: There are a number of traditional medicinal practices that reflect the diversity of Ethiopian cultures. Ethiopian traditional medicine is concerned not only with the curing of diseases but also with the protection and promotion of human physical, spiritual, social, mental and material wellbeing. The many categories of traditional medicinal practices dealing with these different aspects of health include: spiritual healing, prevention, as well as curative and surgical practices. The health and drug policies of the Ethiopian Ministry of Health recognize the important role traditional health systems play in health care. Unfortunately, little has been done in recent decades to enhance and develop the beneficial aspects of traditional medicine including relevant research to explore possibilities for its gradual integration into modern medicine.

Conclusion: The Ethiopian government firmly supports and encourages traditional medicine through its policies as part of the national heritage. Despite these commitments on the policies, the government's ability to implement and provide increased resources for the study, as well as sustainable use of traditional medicine and their integration with modern medical practice has been limited. [Ethiop.J.Health Dev. 2006;20(2):127-134]
\end{abstract}

\section{Introduction}

The World Health Organization (WHO) defines traditional medicine as health practices, approaches, knowledge and beliefs incorporating plant, animal and mineral based medicines, spiritual therapies, manual techniques and exercises, applied singularly or in combination to treat, diagnose and prevent illnesses and maintain well-being (1).

It is known that many countries in African, Asia and Latin America use traditional medicine (TM) to meet some of their primary health care needs. In Africa, up to $80 \%$ of the population uses traditional medicine for primary health care (2).

Traditional medicine has maintained its popularity in all regions of the developing world and its use is rapidly spreading in the industrialized countries. In China, for example, traditional herbal preparations account for $30 \%$ $50 \%$ of the total medicinal consumption. In Ghana, Mali, Nigeria and Zambia, the first line of treatment for $60 \%$ of children with high fever resulting from malaria is the use of herbal medicines at home. WHO estimates that in several African countries traditional birth attendants assist in a majority of births $(2,3)$.

Over one-third of the population in developing countries lack access to essential medicines. The provision of safe and effective TM/CAM therapies could, thus, become a critical tool to increase access to health care (2).

Despite its existence and continued use over many countries, and its popularity and extensive use during the last decade, traditional medicine has not been officially recognized in most countries. Consequently, education, training and research in this area have not been accorded due attention and support. The quantity and quality as well as the safety and efficacy of data on traditional medicine are far from sufficient to meet the criteria needed to support its use worldwide. One of the reasons for the lack of research data is due to health care policies (4).

In Ethiopia up to $80 \%$ of the population uses traditional medicine due to the cultural acceptability of healers and local pharmacopeias, the relatively low cost of traditional medicine and difficult access to modern health facilities. In 2000 only $9.45 \%$ of all deliveries in Ethiopia were attended by trained attendants and health workers. The rest were attended by traditional birth attendants or relatives $(5,6)$.

The vast majority of Ethiopia's population lives in rural areas where the health care coverage is low and where existing public sector resources are being stretched to the limits. One of the greatest challenges facing the country is determining how best to narrow the gap between the

${ }^{1}$ Jimma University, Faculty of Public Health, P.O. Box 5035, E-mail kebededeka@yahoo.com, Jimma, Ethiopia 
existing services and the population whose access to them is very limited (7).

The Ethiopian people's reliance on traditional medicine is also reflected by the fact that Ethiopian migrants in developed countries continue using them. For example, a number of herbs, traditional medical devices and traditional practitioners are available in the UK (8).

In $2003 / 04$, at least $30 \%$ of populations did not have easy access to formal health services. The national average physician to population ratio was $1: 51042$ over $35.1 \%$ of the 1889 physicians working in government and private health services were found in the three big cities accommodating $4.7 \%$ of the total population. The private practitioners also work exclusively in large towns. If it were possible to calculate for rural alone the rates will be very far below the standard (9). A study of pharmaceutical drug use showed that $35 \%$ of the patients did not obtain the prescribed drugs due to lack of money (10). However, most traditional medicines are delivered either free or with a relatively low cost, which contributes to the use of rural based healers for community primary health care need.

The current Ethiopian health care system is primary health care focused. However, traditional healers continue to practice out side of the health services although they are the main health care providers for many Ethiopians $(8,11)$.

Having recognized the significance of traditional medicine more readily, greater attention has been paid by governments of many developing countries in recent years to promote the widespread application of the practice in health care. This has given a new impetus to relevant research, investment and design of programs in the area in many countries (12). In this article, some of the major characteristics and practices of traditional Ethiopian medicine have been summarized in an attempt to provide a brief overview relevant for health policy. Moreover, an attempt is made to review policies regarding traditional medicine towards a better understanding of the historical background and policy perspectives on traditional medicine in Ethiopia.

\section{Search Methods}

Some of the existing extensive literatures on Ethiopian traditional medicine and policy documents were reviewed for this study. Other main sources were books, journals, online materials, reports and other relevant published and unpublished documents. Google, Pub Med and Medline online searches were also carried out to access other relevant publications. In addition, materials from the libraries of Jimma University and the Ethiopian Federal Ministry of Health were reviewed. Several authorities were consulted through personal communication to complement the review of the literature.

The key words used to access online materials include traditional medicine, tradition, and traditional healer with or with out Ethiopia.

\section{Traditional medicine practitioners and practices}

Traditional practitioners include bonesetters birth attendants, tooth extractors, (called 'Wogesha' and yelimd awalaj' respectively in amharic) herbalists, as well as 'debtera', 'tenquay' (witch doctors), and spiritual healers such as 'weqaby' and 'kalicha' $(8,11)$.

Religious practices play a large part in the healing process for Ethiopians such as praying and going to church. Holy water for Orthodox Christians (called 'tsebel' in Amharic) or 'zemzem' in the case of Moslems is also frequently used for a wide varity of illnesses. Ethiopians believe that holy water cures when it is drunk or bathed in $(8,11)$.

\section{Characteristics of traditional medicine in Ethiopia}

Ethiopia has a long history of traditional medicine and has developed ways to combat disease through it (11). The ways are also as diverse as the different cultures. Healing in Ethiopian traditional medicine is not only concerned with curing of diseases but also with the protection and promotion of human physical, spiritual, social, mental and material wellbeing (13).

It is widely believed in Ethiopia that the skill of traditional health practitioners is 'given by God' and knowledge on traditional medicines is passed orally from father to a favorite child, usually a son or is acquired by some spiritual procedures. Traditional Healing knowledge is guarded by certain families or social groups (14).

Healers obtain their drugs mainly from natural substances and in descending order of frequency these constitute plants, animals and minerals. Drugs are prepared in various dosage forms including liquids, ointments, powders and pills. Drugs are also prescribed in a nonformulated form and additives are usually incorporated and more than one drug is used in a single dosage form. Drugs were administered using different routes, the main ones being, topical, oral and respiratory. When sideeffects became severe, antidotes were claimed to be used. The healers imposed restriction when certain types of drugs were taken by patients. Drugs are stored usually in containers such as bottles, papers, pieces of cloth, leaves and horns, and were kept anywhere at home (15).

In addition to traditional medicinal practices by professional healers, there is also an old tradition of self care in the home and another, more recently evolved 
subsystem of lay care of transitional medicine $(16,17)$, which are beyond the scope of this review. There are many types of traditional healing practices, which are discussed here in four groups.

In Ethiopian traditional medicine, the issue of health is seen holistically and not separated into physical health and mental health. Spiritual wellbeing is also important. Health is seen as a 'gift of God' or 'the will of God' and many Ethiopians generally believe that their religion helps keep them healthy (8).

These perceptions are related to the belief that supernatural forces are involved in causing disease as well as in their treatment. For instance, while the devil is considered to be the cause of a number of illnesses, God is believed to provide the healing. This scenario is even more applicable to mental illnesses, and some other conditions that are more obscure and difficult to treat with well-defined means. The use of prayer as a therapy in the practice to a supernatural power has been around for a much longer time in the practice of Ethiopian traditional medicine. Traditional spiritual healers are known by different names, depending on the communities where they practice including debtera, tenquay (witch doctors), weqaby and kalicha $(8,11,18)$.

The more widely known spiritual healers are grouped into two categories according to their religious beliefs. Members of the Orthodox Christian clergy are called the debteras and members of the Muslim community are known as kalichas. Debteras usually look upon mental disorders as possession by evil spirits, which are thus treated mostly by praying and using holy water or eventually exorcising the evil spirit. The debteras are renowned for their prayer which is known as degmit, which they perform on behalf of clients. Along with this, they prepare holy water (tsebel) and kitab amulets containing a written script. By means of the degmit, the debteras claim to have the ability to perform miracles, which are believed to be manifested by the reactions of their patients. Tsebel is commonly used to ward off evil spirits from patients who are believed to be possessed by the devil. Kitabs are worn for the purpose of protecting oneself against the evil eye or buda, as well as snake and scorpion bites.

The kalichas is the religious leader who, through an ecstatic ritual, can investigate the causes of a disorder and advise the patient on what to do. Mental disorders are generally explained as resulting from disturbances in the relationship between people and divinity. They conduct special ceremonies to effectively perform their practices which include the use of excessive smoke by burning incense such as myrrh, and frankincense (etan). Similar to the debteras, the kalichas also prepare kitabs of their own kind to be worn by their clients (18).
Besides its role in healing, traditional Ethiopian medicine is also associated with a number of other beliefs. These practices are helpful in prevent the evil eye, overcoming demons, defeating human enemies, obtaining long life, clear eye sight, a good memory, as well as a large family and a faithful wife (11).

\section{Preventative practice}

Traditional Ethiopian medicine includes several elements or disease prevention. In the past, the spread of highly infectious diseases such as smallpox was prevented by deserting places where the epidemics occurred. Moreover, people were inoculated by taking pus from a sick person during special rituals (19). Sweeping or covering floors with particular plants is another traditionally practiced disease preventive measure. Other methods of disease prevention include isolating people with contagious diseases, prohibition or controlling movement and taking children away from the affected areas $(11,19,20)$.

As noted above, kitabs are also used for the purpose of protecting an individual against evil eye, as well as snake and scorpion bites. Amulets, arm rings, hair style and eye make-up (antimony or kool) are also supposed to protect from the evil eye. Still other medicines are available for use as charms against an enemy. In addition, cultural rituals and scarification are commonly employed in diseases prevention (21).

\section{Curative practices}

Traditional Ethiopian medicine is commonly used to treat a variety of diseases employing substances as recommended by professional traditional medical practitioners. The conditions that claim to be treated include gastrointestinal disturbances, respiratory disorders, sexually transmitted infections, tuberculosis, impotency, hemorrhoids, rabies, intestinal parasites, skin problems, liver diseases, mental disorders, hypertension, diabetes, gynecological conditions rheumatism, malaria and others (11). Professional traditional healers known by different names in different parts of the country are the primary players in the curative aspect of traditional medicine practice. One of the well recognized groups of these healers are the secular medhanit awakis (kitel betashs) herbalists using plants as their primary means of providing treatment. A large number of plant medicines are used, and for the purpose of references, most medhanit awakis possess pharmacopoeias. Minerals and animal-derived substances are additional items in the pharmacopoeia of medhanit awakis (22).

The medhanit awakis diagnose disease conditions mostly by physical examination and questioning patients. Sometimes they prescribe medicines based on descriptions from informants. They prepare their medicines in various dosages and forms and administer medicines via different routes. After preparations, many 
of the medicines are stored in containers anywhere at home without special requirements. Although the medhanit awakis make efforts to modernize their practices, they do not normally employ any of the equipment and techniques used in conventional medical or pharmaceutical practices (22).

\section{Surgical practice}

Traditional practices considered to be related to surgery include bone-setting, uvulectomy, circumcisions, bleeding and cupping, cautery, scarification and tooth extraction $(22,23)$. The setting of bones is regarded as an important surgical procedure which requires a certain degree of skill and experience on the part of the healer. In most places, the healer involved in bone-setting is the local wogesha. In many situations, the wogesha practices his/her skills without aseptic conditions, with or without the application of medicines. Other procedures are indicated for more specific conditions such as rheumatism, bleedings, swelling, wounds, headache, localized infections, and snake and scorpion bites (11).

Midwifery is one of the most common practices of traditional Ethiopian medicine. It is performed by traditional midwives commonly known in Amharic as yelmid awalajs, (traditional birth attendants) and most yelmid awalajs are women. Depending on the need, they carry out their practice with or without the administration of medicines. During the Derg regime, some 11,500 Yelmid awalajs were trained for better utilization of their knowledge in midwifery (14).

Traditional medicines are sold in every open market in Ethiopia and households, especially in the rural areas. Market vendors selling these materials are women. These medicines are usually sold to the public together with other materials such as spices, salt and other food items (24).

Traditional medical treatments are also commonly given at the household level. There is significant knowledge of medicinal plants in the non professional public domain where most ailments are diagnosed and treated at the household level. Where traditional professionals are consulted, it is often for their specialized traditional knowledge and skills pertaining to a relatively limited range of health. Most of the families grow or gather these plants in their vicinities of homes (17).

\section{Problems associated with traditional medicine}

Some plants used in traditional medicines, such as taenicides, are widely known to be toxic. For example, blindness and changes in central nervous system function have repeatedly been found in people who took over dosage of Hagenia abyssinica (25). Traditional healers may cause create delays in the treatment of communicable diseases such as TB if they fail to refer patients to modern health services (26). Moreover, a number of harmful practices have been traced to healers, including female genital mutilation, uvulectomy, and milk tooth extraction (27).

\section{Review of Policies}

Formal recognition to TM in Ethiopia was given in 1942 (Proc. 27) where the legality of the practice is acknowledged as long as it does not have negative impact on health. This was reaffirmed in the 1943 and 1948 (Proc. 100) Medical Registration Proclamations. Articles in the Ethiopian Penal Code (512/1957) and the Civil Code (8/1967) provide guidelines for the practice of traditional medicine. But they did not stipulate any requirement for registration. Registration and licensing was introduced in $1950(13,28)$. During the Derg period of the 1970s and 1980s the country's health policy emphasized disease prevention and health service development in the rural areas. It was followed by official attention to the promotion and development of traditional medicine, particularly after the adoption of the Primary Health Care Strategy in 1978. In November 1979, the Office for the Coordination of Traditional Medicine was established. It conducted chemical assays and biomedical studies of some herbal medicines and a total of 6,000 traditional practitioners were registered and a monograph describing 260 medicinal plants was prepared. Meetings and workshops were organized that brought together traditional and modern medical practitioners. In different areas of the country healers also formed their own professional associations. However, these lacked guidance, funds and personnel to help them move forward (14, 28, and 29).

The National Policy of Traditional Medicine under the current Federal Democratic Republic of Ethiopia was issued as part of the Health, Drug, and Science and Technology Policy issued in 1993 (30,31,32). Traditional medicine is placed as one of the eight priorities of the current Health Policy. It was reported that due attention shall be given to the development of the beneficial aspects of traditional medicine including related research and its gradual integration into Modern Medicine. The general strategies adopted include identifying and encouraging the utilization of its beneficial components, coordinating and encouraging research including its linkage with modern medicine and developing appropriate regulation and registration of practitioners (30).

The 10 targets of the drug policy include conducting coordinated research on traditional medicines and striving for development into pharmaceutical drugs. The same document outlines general strategies for strengthening the health sector through research and development, creating favorable conditions for the development of safe and effective drugs and involving private providers (31). 
Laws and regulations on traditional medicine were issued under the Drug Administration and Control Proclamation No. 176/99. The national drug program is one of the responsibilities of the Drug Administration and Control Authority (DACA) which was established by Proclamation No. 176.DACA. An Expert Committee is also a part of DACA. DACA is responsible for preparing standards of safety, efficacy and quality of traditional medicine, and shall evaluate laboratory and clinical studies. It also gives license for the use of traditional medicine in the official health services. The national research institute covering both traditional and herbal medicine is the Drug Research Department of the Ethiopian Health and Nutrition Research Institute (EHNRI) (12).

Currently there is no registered traditional practitioner and way of registration in the Federal Ministry of Health (33) though herbal medicines are sold on the streets with medical claims. No regulatory requirements exist for the manufacturing or safety assessment of traditional medicines and herbal medicines are not included in the essential medicines list. There is neither a post market surveillance system, a restriction on the sale of herbal medicines nor a guideline for clinical trials using traditional medicines (12).

An initiative was made to establish guidelines for licensing and minimum standards for traditional practice and practitioners. A committee from FMOH, DACA and EHNRI is preparing standards of safety, efficacy and the quality of traditional medicine (33). Different researches were also conducted on the toxicology and efficacy of drugs in the Drug Research Department of the EHNRI (34).

By early 2006 DACA had not yet carried out any activity on traditional medicines and no traditional drug was registered and licensed although guidelines are being prepared (35).

Similarly, there is no training program exists on traditional medicine and there is no guideline for training traditional healers.

The Ethiopian Intellectual Property (IP) office with funds provided by the World Bank is preparing an intellectual property protection policy for traditional medicine (36).

\section{Discussion}

Due to poor access to health services, especially in the rural areas, the majority of the Ethiopia people rely mainly on traditional medicine for their primary health care needs (5). Nevertheless, the system has been neglected and its therapeutic potentials as well as adverse effects have not been thoroughly studied scientifically. Moreover, the integration of the practice of traditional medicine into the formal health system has not been seriously considered. Progress made so far in this direction in other countries has allowed for a wide utilization of traditional medicine and better recognition of its practitioners as heritage benefiting the majority of their people (37).

The interest in and intrinsic value of traditional Ethiopian medicine should not be only be attributed to the lack of access to modern medicinal services. Even in cites where modern health services are more accessible and specialized, many people continue to go to traditional healers $(6,14)$. This may be due to the cultural acceptability of healers, the respect they have and their easy accessibility to clients. The healing process is often carried out in an atmosphere of mutual confidence and shared expectations. Compensation in traditional medicine is different from the payment system of official health services. Healers typically receive an initial payment in money (or in goods in rural areas) and then negotiate subsequent payments with the patient. In cases, where treatment is ineffective, the patient does not have to pay beyond the initial payment (38).

As mentioned above, in spite of its potentials for the development and greater contribution, traditional medicine in Ethiopia has not received significant inputs from the administration. This is more revealing when compared with what has been achieved in many other developing countries. The system is deteriorating primarily due to the lack of concern and, at times, due to apparent ignorance on the part of the authorities in responsible positions (14). Lack of concern goes far beyond the areas of natural resources conservation.

Environmental degradation, deforestation, agricultural expansion, over grazing and high population growth are potential treats to the survival of many potential valuable medicinal plants (5). This trend is increasingly jeopardizing the ethno botanical and other natural resources of the country, especially in the highlands, largely due to over population, inadequate environmental policies and failure to fully implement them $(39,10)$.

All healing practices involving traditional medicines in Ethiopia continue to be limited to the non-official sectors. This fact alone contributes to isolation and subsequent non-utilization of this TM in the national health system. The official Ethiopian health care system is almost exclusively based on the concept of modern medicine and the man power employed generally does not have adequate knowledge of the traditional healing systems. However, efforts were recently made by the Medical Faculty of Addis Ababa University to expand its curriculum and encourage research on TM. Hopefully, these and similar efforts by international organizations such as WHO (41), will help to change prevailing attitudes. There is also a need to change the widely held notion by the national and international medical 
community that biomedical research is generally more significant and prestigious than that devoted to local health systems. The relatively small amount of research devoted to indigenous medicine and health care systems is striking in view of its importance in meeting the health care needs of large populations who tend to be afflicted with most acute health problems.

In the absence of a clear indication on the legal status and benefits emanating from their healing practices, traditional health practitioners would find it very difficult to part their knowledge to researchers whom they do not trust. From the side of modern practitioners, very little effort has been made to understand traditional healing practitioners. Most modern health professionals in Ethiopia seem to think that there is no logic to what ever traditional healing practitioners do. This attitude contributes to the continuation of polarization and separate development of the two systems with out creating a basis for integration.

Policies conductive to TM exist in Ethiopia and compared to the former regimes, the current policy is comprehensive and gives TM an important place.

In spite of the promulgation of the necessary policies, little has been done in recent decades to enhance and develop the beneficial aspects of traditional medicine including related research and its gradual integration into modern medicine (5). There are many gaps between policy and actual practices. There are clearly deficits in the organized approach towards ensuring an optimal contribution of TM to the national health system. For example, there were no regulations to the safety and efficacy, licensing, as well as the registration and guidelines for clinical trails involving traditional medicines (33-35).

Moreover, there is no training institute exist on traditional medicine. It is well known that trained healers learn new knowledge quickly and integrate it in to their practices. Training of adequate modern health professionals can also provide better understanding of the traditional system. This acquired knowledge by the professionals in the two systems of medicine may lead to mutual respect, mutual understanding, productive collaboration (42) and delivery of effective health services.

There is evidence suggesting a declining trend in the number of traditional resources of medicinal plants (5). This calls for an urgent action to document and preserve the traditional medical knowledge before it disappears from the country.

\section{Conclusion}

The Ethiopian government firmly supports and encourages traditional medicine as part of the national heritage. Policies and mandates are clearly given to different government ministries and authorities. However, there are several gaps at the policy level, and only a very limited number of efforts have been made in recent years in implementing them at the community level.

Thus, any organizational effort must be directed to an integrative plan that includes manpower planning, the development of training programs the issuance of regulations on safety and efficacy, the licensing, and guidelines for clinical trail of traditional medicines. This should include the determination of the legal status of traditional practitioners, including clearly stated benefits they could obtain from closer collaboration as well as their responsibilities. Moreover there is a need to involve traditional healers on all the aspects of traditional medicine, including the preparation of pharmacopoeia for evaluating all medical plants and other substances used in drugs, giving therapeutic indicators, and methods of preparations and dosages, as well as awards of grants for research on traditional medicine in collaboration with other interested institutions and organizations and harmonization between traditional and modern medicine. In order to improve the prospects and potential benefits of traditional medicine to society, the contribution of both health care systems needs to be considered.

\section{Acknowledgement}

Our heart felt gratitude goes to Prof. Challi Jira, Jimma University Department of Health Planning and Management for his critical reading and invaluable comments. We are also grateful to staffs of federal Ministry of Health Library for their assistance in searching documents.

\section{References}

1. WHO. Legal Status of Traditional Medicine and Complementary/Alternative Medicine: A World Wide Review. Geneva 2001.

2. WHO. Fact Sheet, Traditional Medicine, Geneva, May 2003.

3. Bannerman RH, Burton J and Chien W. Traditional Medicine and Health Care Coverage. World health Organization, Geneva, Switzerland 1993.

4. WHO. General Guidelines for Methodologies on Research and Evaluation of Traditional Medicine WHO/EDM/TRM/2000. 1, Geneva, Switzerland 2000.

5. Lambert J. Ethiopia: Traditional medicine and the bridge to better health. World Bank, available at: http://www.worldBank.rog/afr/ik/dfefault.htm.

6. Central Statistical Authority and ORC Macro Ethiopia Demographic and Health Survey 2000. Addis Ababa Ethiopia and Calverton, Maryland, USA, May 2001.

7. Transitional Government of Ethiopia Health Sector Strategy. Addis Ababa, April 1995. 
8. Papadopoulos R, Lay M, Gebrehiwot A. Cultural snapshots: A guide to Ethiopian refugees for health care workers. Research Center for Trans-cultural Studies in Health, Middlesex University, London UK. N 14 4YZ, May 2002. Available at: www.mdx.ac.uk/www/rctsh/embrace.htm.

9. Federal Democratic Republic of Ethiopia Ministry of Health. Health and Health Related Indicators 2003/04. Addis Ababa, December 2004.

10. Federal Democratic Republic of Ethiopia, Ministry of Health. Assessment of the Pharmaceutical Sector in Ethiopia. Addis Ababa, October 2003.

11. Negussie B. Traditional wisdom and modern development: A case study of traditional peri-natal knowledge among women in southern Shewa, Ethiopia. Doctoral dissertation, University of Stockholm, December 1988.

12. WHO. National Policy on Traditional Medicine and regulation of Herbal medicines, Report of a WHO Global Survey, Geneva, Switzerland May 2005.

13. Beshaw M. Promoting traditional medicine in Ethiopia: A brief historical overview of government Policy. Soc. Sci and Med, 1991;33:193-200.

14. WHO. Traditional Medicine Program and Global program on AIDS. Report on the Consultation on AIDS and Traditional Medicine on WHO meeting (Unpublished WHO/TRM/GPA/90.1) Francis town, Botswana 23-27 July 1990.

15. Abebe W. Traditional pharmaceutical practices in Gondar Region, northwestern Ethiopia. Ethnopharmacol 1984;11)1):33-47.

16. Slikkerveer LJ. Plural medical system in the Horn of Africa: The legacy of "Sheikh" Hippoates 1990 London: Kegan Paul.

17. Fassil H. "We do what we know". Local health knowledge and home-based medicinal plant use in Ethiopia. Doctoral dissertation, International Development Center, Oxford University, 2003.

18. Jacobsson L, Merdasa F. Traditional perceptions and treatment of mental disorders in western Ethiopia before the 1974 revolution. Acta Psychiatr Scand 1991;84(5):475-81.

19. Pankhurst R. The history and traditional treatment of smallpox in Ethiopia. Med Hist 1965;9:343-346.

20. Pankhurst R. The history of cholera in Ethiopia. Med Hist 1968;12:262-268.

21. Schneider P, Shewangizaw E, Taye E, Gebrehiwot T, Worku S, Oppermann J, Leupold W, Teka T. Traditional medicine in Ethiopia in childhood diseases. Kinderarztl Praxis 1989;57(8):393-9.

22. Moges A. Traditional Ethiopian medicine. Conference paper for the Eighth International Conference of Ethiopia Studies. Addis Ababa 1984.

23. Gebere Selassie, Desta M, Negesh Z. Harmful traditional practices affecting the health of women and children in Ethiopia. Ministry of Health/UNICEF, Addis Ababa, Ethiopia. 1984.
24. Kloos H, Tekle A, Yohannes L, Yosef A, Lemma A. Preliminary studies of traditional medical plants in nineteen markets in Ethiopia: Use patterns and public health aspects. Ethiopia Med J. 1978;16(2): 33-43.

25. Rokos L. Eye complications in poisoning caused by "Kosso" (Hagenia abyssinica). Ethiop Med J 1969;7:11.

26. Yimer s, Bijune G, Alene G. Diagnostic and treatment delay among pulmonary tuberculosis patients in Ethiopia. A cross sectional study. BMC infectious Diseases 2005;5:112.

27. National Committee on Traditional Practices of Ethiopia. Project Report: Baseline Survey on Harmful Traditional Practices in Ethiopia. Addis Ababa, Ethiopia. February 1998.

28. Ministry of Health. History of Ethiopian Health Service. Addis Ababa, Ethiopia. January 1984.

29. The Transitional Government of Ethiopia Office of the Council of Ministers. Report of the National Health Policy Task Force, Social and Administration Affairs. Addis Ababa, Ethiopia. February 1993.

30. Ministry of Health. Health Policy of the Transitional Government of Ethiopia, Addis Ababa, Ethiopia. Sept. 1993.

31. Ministry of Health. National Drug Policy of the Transitional Government of Ethiopia, Addis Ababa, Ethiopia. November 1993.

32. Transitional Government of Ethiopia, Science and Technology Commission, National Policy on Science and Technology, Addis Ababa, Ethiopia. December 1993.

33. Amensisa J. Personal Communication 18 January 2006 Ethiopian Federal Ministry of Health, Department of Health Service and Training, health Service team leader, Addis Ababa, Ethiopia.

34. Urga K. Personal Communication. 18 January 2006 Ethiopian Health and Nutrition Institute, Head of the Department Drug Research, Addis Ababa, Ethiopia.

35. Feleke W. Personal Communication. 18 January 2006 Drug Administration and Control Authority (DACA) Registration Expert, Addis Ababa, Ethiopia.

36. The Ethiopian Herald. I.P. to formulate property protection policy. 30 March 2005.

37. WHO. The role of traditional medicine in primary health care in China. WHO, Geneva, Switzerland, 1985.

38. Leonard KL. African traditional healers: The economics of healing. World Bank, available at: http://www.worldbank.org/afr/ik/default.htm.

39. Feoli E, Vuerich LG, Woldu Z. Evaluation of environmental degradation in northern Ethiopia using GIS to integrate vegetation, geographical erosion and socio-economic factors. Agriculture, Ecosystems and Environments 2002;91;313-325.

40. Jabbar M, Pender J, Ehui S (eds.). Policies for sustainable land management in the highlands of 
Ethiopia. Socio Economic and Policy Research Working paper No. 30, International Livestock Research Institute, Addis Ababa, Ethiopia, 2000.

41. WHO. WHO Traditional Medicine Strategy 20022005. World Health Organization, Geneva, Switzerland. 2002.
42. Dikasso D, Urga K, Addis G, Tadele A. The attitude of modern health workers towards traditional health care system in Wolayita Zone. J Ethiop Med Pract 2002;4(2):92.99. 